\title{
A Multigrid Block Krylov Subspace Spectral Method for Variable-Coefficient Elliptic PDE
}

\author{
James V. Lambers \\ Department of Mathematics, University of Southern Mississippi, 118 College Dr \#5045, \\ Hattiesburg, MS 39406-0001 USA
}

\begin{abstract}
Krylov subspace spectral (KSS) methods have been demonstrated to be effective tools for solving time-dependent variable-coefficient PDE. They employ techniques developed by Golub and Meurant for computing elements of functions of matrices to approximate each Fourier coefficient of the solution using a Gaussian quadrature rule that is tailored to that coefficient. In this paper, we apply this same approach to time-independent PDE of the form $L u=g$, where $L$ is an elliptic differential operator. Numerical results demonstrate the effectiveness of this approach, in conjunction with residual correction applied on progressively finer grids, for Poisson's equation and the Helmholtz equation.
\end{abstract}

Keywords: spectral methods, Gaussian quadrature, block Lanczos method, Poisson's equation, Helmholtz equation

PACS: 02.60.Lj, 02.60.Dc, 02.60.Jh

\section{INTRODUCTION}

Let $L$ be an elliptic second-order differential operator of the form

$$
L u=-\nabla \cdot(p \nabla u)+q u,
$$

where $p(x, y)>0$ and $q(x, y)$ are smooth functions. We consider the following boundary value problem on a rectangle,

$$
L u=g(x, y), \quad 0<x, y<2 \pi,
$$

with homogeneous Dirichlet boundary conditions, or periodic boundary conditions.

In [15] a class of methods, called Krylov subspace spectral (KSS) methods, was introduced for the purpose of solving parabolic variable-coefficient PDE. These methods are based on techniques developed by Golub and Meurant in [5] for approximating elements of a function of a matrix by Gaussian quadrature in the spectral domain. In $[8,11]$, these methods were generalized to the second-order wave equation, for which these methods have exhibited even higher-order accuracy.

It has been shown in these references that KSS methods, by employing different approximations of the solution operator for each Fourier coefficient of the solution, achieve higher-order accuracy in time than other Krylov subspace methods (see, for example, [9]) for stiff systems of ODE, and, as shown in [11], they are also quite stable, considering that they are explicit methods. In [12, 13], the accuracy and robustness of KSS methods were enhanced using block Gaussian quadrature. Recent extensions include the time-dependent Schrödinger equation [14] and Maxwell's equations [17]. 
It is our belief that by a change of integrand in the integrals used to compute the Fourier coefficients of the solution, the high accuracy achieved for time-dependent problems can be extended to the time-independent case, even for cases in which the operator $L$ is indefinite, as in the Helmholtz equation. In this paper, we will see that by applying block KSS methods in conjunction with residual correction, first on coarser grids and proceeding to finer grids, we do in fact obtain a highly accurate and efficient method for these problems. Section 2 reviews the main properties of KSS methods, including block KSS methods, and explains how they can be applied to elliptic problems. Numerical results are presented in Section 3, and conclusions are stated in Section 4.

\section{KRYLOV SUBSPACE SPECTRAL METHODS}

We first review KSS methods, which were first developed in [15] for parabolic problems. Let $S=L^{-1}$ represent the exact solution operator of the problem (2), restricted to one space dimension for simplicity, and let $\langle\cdot, \cdot\rangle$ denote the standard inner product of functions defined on $[0,2 \pi]$,

$$
\langle u(x), v(x)\rangle=\int_{0}^{2 \pi} \overline{u(x)} v(x) d x .
$$

Krylov subspace spectral methods, introduced in [15], use Gaussian quadrature on the spectral domain to compute the Fourier coefficients of the solution. Given the right-hand side $g(x)$, the solution is computed by approximating the Fourier coefficients that would be obtained by applying the exact solution operator to $g(x)$,

$$
\hat{u}(\omega)=\left\langle\frac{1}{\sqrt{2 \pi}} e^{i \omega x}, S g(x)\right\rangle .
$$

\section{Elements of Functions of Matrices}

In [5] Golub and Meurant describe a method for computing quantities of the form

$$
\mathbf{u}^{T} f(A) \mathbf{v},
$$

where $\mathbf{u}$ and $\mathbf{v}$ are $N$-vectors, $A$ is an $N \times N$ symmetric positive definite matrix, and $f$ is a smooth function. Our goal is to apply this method with $A=L_{N}$ where $L_{N}$ is a spectral discretization of $L, f(\lambda)=\lambda^{-1}$, and the vectors $\mathbf{u}$ and $\mathbf{v}$ are derived from $\hat{\mathbf{e}}_{\omega}$ and $\mathbf{g}$, where $\hat{\mathbf{e}}_{\omega}$ is a discretization of $\frac{1}{\sqrt{2 \pi}} e^{i \omega x}$ and $\mathbf{g}$ represents the right-hand side function $g(x)$, evaluated on an $N$-point uniform grid.

The basic idea is as follows: since the matrix $A$ is symmetric positive definite, it has real eigenvalues

$$
b=\lambda_{1} \geq \lambda_{2} \geq \cdots \geq \lambda_{N}=a>0
$$


and corresponding orthogonal eigenvectors $\mathbf{q}_{j}, j=1, \ldots, N$. Therefore, the quantity (5) can be rewritten as

$$
\mathbf{u}^{T} f(A) \mathbf{v}=\sum_{j=1}^{N} f\left(\lambda_{j}\right) \mathbf{u}^{T} \mathbf{q}_{j} \mathbf{q}_{j}^{T} \mathbf{v} .
$$

We let $a=\lambda_{N}$ be the smallest eigenvalue, $b=\lambda_{1}$ be the largest eigenvalue, and define the measure $\alpha(\lambda)$ by

$$
\alpha(\lambda)= \begin{cases}0, & \text { if } \lambda<a \\ \sum_{j=i}^{N} \alpha_{j} \beta_{j}, & \text { if } \lambda_{i} \leq \lambda<\lambda_{i-1} \\ \sum_{j=1}^{N} \alpha_{j} \beta_{j}, & \text { if } b \leq \lambda\end{cases}
$$

where $\alpha_{j}=\mathbf{u}^{T} \mathbf{q}_{j}$ and $\beta_{j}=\mathbf{q}_{j}^{T} \mathbf{v}$. If this measure is positive and increasing, then the quantity (5) can be viewed as a Riemann-Stieltjes integral

$$
\mathbf{u}^{T} f(A) \mathbf{v}=I[f]=\int_{a}^{b} f(\lambda) d \alpha(\lambda) .
$$

As discussed in [5], the integral $I[f]$ can be approximated using Gaussian quadrature rules, which yield an approximation of the form

$$
I[f]=\sum_{j=1}^{K} w_{j} f\left(t_{j}\right)+R[f],
$$

where the nodes $t_{j}, j=1, \ldots, K$, as well as the weights $w_{j}, j=1, \ldots, K$, can be obtained using the symmetric Lanczos algorithm if $\mathbf{u}=\mathbf{v}$, and the unsymmetric Lanczos algorithm if $\mathbf{u} \neq \mathbf{v}$ (see [7]).

\section{Block Gaussian Quadrature}

In the case $\mathbf{u} \neq \mathbf{v}$, there is the possibility that the weights may not be positive, which destabilizes the quadrature rule (see [1] for details). One option to get around this problem is rewriting (5) using decompositions such as

$$
\mathbf{u}^{T} f(A) \mathbf{v}=\frac{1}{\delta}\left[\mathbf{u}^{T} f(A)(\mathbf{u}+\delta \mathbf{v})-\mathbf{u}^{T} f(A) \mathbf{u}\right],
$$

where $\delta$ is a small constant. Guidelines for choosing an appropriate value for $\delta$ can be found in [15, Section 2.2].

If we compute (5) using (11) or the polar decomposition

$$
\frac{1}{4}\left[(\mathbf{u}+\mathbf{v})^{T} f(A)(\mathbf{u}+\mathbf{v})-(\mathbf{v}-\mathbf{u})^{T} f(A)(\mathbf{v}-\mathbf{u})\right],
$$

then we have to carry out the process for approximating an expression of the form (5) with two sets of starting vectors, whereas a single quadrature rule is more desirable. Instead, we consider

$$
\left[\begin{array}{ll}
\mathbf{u} & \mathbf{v}
\end{array}\right]^{T} f(A)\left[\begin{array}{ll}
\mathbf{u} & \mathbf{v}
\end{array}\right]
$$


which results in the $2 \times 2$ matrix

$$
\int_{a}^{b} f(\lambda) d \mu(\lambda)=\left[\begin{array}{ll}
\mathbf{u}^{T} f(A) \mathbf{u} & \mathbf{u}^{T} f(A) \mathbf{v} \\
\mathbf{v}^{T} f(A) \mathbf{u} & \mathbf{v}^{T} f(A) \mathbf{v}
\end{array}\right]
$$

where $\mu(\lambda)$ is a $2 \times 2$ matrix function of $\lambda$, each entry of which is a measure of the form $\alpha(\lambda)$ from (8).

In [5] Golub and Meurant show how a block approach can be used to generate quadrature formulas. We will describe this process here in more detail. The integral $\int_{a}^{b} f(\lambda) d \mu(\lambda)$ is now a $2 \times 2$ symmetric matrix and the most general $K$-node quadrature formula is of the form

$$
\int_{a}^{b} f(\lambda) d \mu(\lambda)=\sum_{j=1}^{K} W_{j} f\left(T_{j}\right) W_{j}+\text { error }
$$

with $T_{j}$ and $W_{j}$ being symmetric $2 \times 2$ matrices. By diagonalizing each $T_{j}$, we obtain the simpler formula

$$
\int_{a}^{b} f(\lambda) d \mu(\lambda)=\sum_{j=1}^{2 K} f\left(\lambda_{j}\right) \mathbf{v}_{j} \mathbf{v}_{j}^{T}+\text { error }
$$

where, for each $j, \lambda_{j}$ is a scalar and $\mathbf{v}_{j}$ is a 2-vector.

Each node $\lambda_{j}$ is an eigenvalue of the matrix

$$
\mathscr{T}_{K}=\left[\begin{array}{ccccc}
M_{1} & B_{1}^{T} & & & \\
B_{1} & M_{2} & B_{2}^{T} & & \\
& \ddots & \ddots & \ddots & \\
& & B_{K-2} & M_{K-1} & B_{K-1}^{T} \\
& & & B_{K-1} & M_{K}
\end{array}\right]
$$

which is a block-triangular matrix of order $2 K$. The vector $\mathbf{v}_{j}$ consists of the first two elements of the corresponding normalized eigenvector.

To compute the matrices $M_{j}$ and $B_{j}$, we use the block Lanczos algorithm, which was proposed by Golub and Underwood in [6]. Let $X_{0}$ be an $N \times 2$ given matrix, such that $X_{1}^{T} X_{1}=I_{2}$. Let $X_{0}=0$ be an $N \times 2$ matrix. Then, for $j=1, \ldots, K$, we compute

$$
\begin{gathered}
M_{j}=X_{j}^{T} A X_{j}, \\
R_{j}=A X_{j}-X_{j} M_{j}-X_{j-1} B_{j-1}^{T}, \\
X_{j+1} B_{j}=R_{j} .
\end{gathered}
$$

The last step of the algorithm is the $Q R$ decomposition of $R_{j}$ such that $X_{j+1}$ is $n \times 2$ with $X_{j+1}^{T} X_{j+1}=I_{2}$. The matrix $B_{j}$ is $2 \times 2$ and upper triangular. The other coefficient matrix $M_{j}$ is $2 \times 2$ and symmetric. 


\section{Block KSS Methods}

We are now ready to describe block KSS methods for elliptic PDE in 1-D of the form $L u=g$. For each wave number $\omega=-N / 2+1, \ldots, N / 2$, we define

$$
R_{0}(\omega)=\left[\begin{array}{ll}
\hat{\mathbf{e}}_{\omega} & \mathbf{g}
\end{array}\right]
$$

and compute the $Q R$ factorization $R_{0}(\omega)=X_{1}(\omega) B_{0}(\omega)$. We then carry out the block Lanczos iteration described in (17) to obtain a block tridiagonal matrix $\mathscr{T}_{K}(\omega)$ of the form (16), where each entry is a function of $\omega$.

Then, we can express each Fourier coefficient of the approximate solution as

$$
[\hat{\mathbf{u}}]_{\omega}=\left[B_{0}^{H} E_{12}^{H}\left[\mathscr{T}_{K}(\omega)\right]^{-1} E_{12} B_{0}\right]_{12}
$$

where $E_{12}=\left[\begin{array}{ll}\mathbf{e}_{1} & \mathbf{e}_{2}\end{array}\right]$. The computation of (18) consists of computing the eigenvalues and eigenvectors of $\mathscr{T}_{K}(\omega)$ in order to obtain the nodes and weights for Gaussian quadrature, as described earlier.

Once the approximation $\mathbf{u}$ is computed using the inverse FFT, we can compute the residual $\mathbf{r}=\mathbf{g}-L_{N} \mathbf{u}$, and correct the solution by applying the block KSS method again to the problem $L_{N} \mathbf{c}=\mathbf{r}$, and updating the solution by $\mathbf{u}=\mathbf{u}+\mathbf{c}$. We can continue this process of residual correction until the residual is sufficiently small.

\section{Implementation}

In [16], it was demonstrated that recursion coefficients for all wave numbers $\omega=$ $-N / 2+1, \ldots, N / 2$ can be computed simultaneously, by regarding them as functions of $\omega$ and using symbolic calculus to apply differential operators analytically, as much as possible. As a result, KSS methods require $O(N \log N)$ floating-point operations. The same approach can be applied to block KSS methods. For both types of methods, it can be shown that for a $K$-node Gaussian rule or block Gaussian rule, $K$ applications of the operator $L_{N}$ to the right-hand side $\mathbf{g}$ are needed. Although we have restricted ourselves to one space dimension in the description of block KSS methods, generalization to higher dimensions is straightforward, as discussed in [16].

\section{NUMERICAL RESULTS}

In this section we demonstrate the effectiveness of block KSS methods for solving elliptic PDE.

\section{Poisson's Equation}

We first apply a 2-node block KSS method to the problem

$$
\nabla \cdot(p(x, y) \nabla u(x, y))=g(x, y), \quad 0<x, y<2 \pi,
$$


with Dirichlet boundary conditions, where

$$
\begin{aligned}
p(x, y) \approx & 4.03+0.017 \cos y+0.0052 \sin y+0.0026 \cos 2 y+0.029 \cos x+ \\
& 0.014 \sin x+0.0083 \cos (x+y)+0.0019 \cos (x-2 y)+ \\
& 0.0073 \cos (x-y)+0.0046 \sin (x-y)+0.0021 \cos 2 x \\
g(x, y) \approx & -2.39 \sin y+1.44 \sin 2 y+0.47 \sin 3 y-0.31 \sin x- \\
& 1.44 \sin (x+y)+0.19 \sin (x+2 y)-5.73 \sin (x-y)-0.53 \sin 2 x- \\
& 0.35 \sin (2 x+y)-1.63 \sin (2 x-y)+1.07 \sin 3 x+ \\
& 0.6 \sin (3 x+y) .
\end{aligned}
$$

The coefficient $p(x, y)$ is constructed so as to have the smoothness of a function with four continuous derivatives with respect to either $x$ or $y$, using a technique described in [15] that imposes a chosen decay rate on Fourier coefficients. The function $f$ is obtained by applying the spatial operator $L u=-\nabla \cdot(p \nabla u)$ to a function $u(x, y)$ that is constructed in the same way as $p(x, y)$, with the same smoothness, so that the exact solution is known.

In our experiments, we will use different grid spacings in order to investigate how the error varies with increasing resolution. The problem data is computed on the finest grid, and projected onto the coarser grids. However, in order to isolate error due to KSS methods themselves, we do not include error due to truncation of Fourier series in our error estimates.

The results are shown in Figure 1 and Table 1. The relative error is rapidly reduced by residual correction until it is not much greater than machine precision. As shown in the figure, we achieve linear convergence, with a very small asymptotic error constant. We also see that the error only increases by a factor of 3 as the number of grid points per dimension doubles, but these error estimates do not include truncation of Fourier series. As will be seen in later experiments, the overall error decreases as the number of grid points increases, as expected.

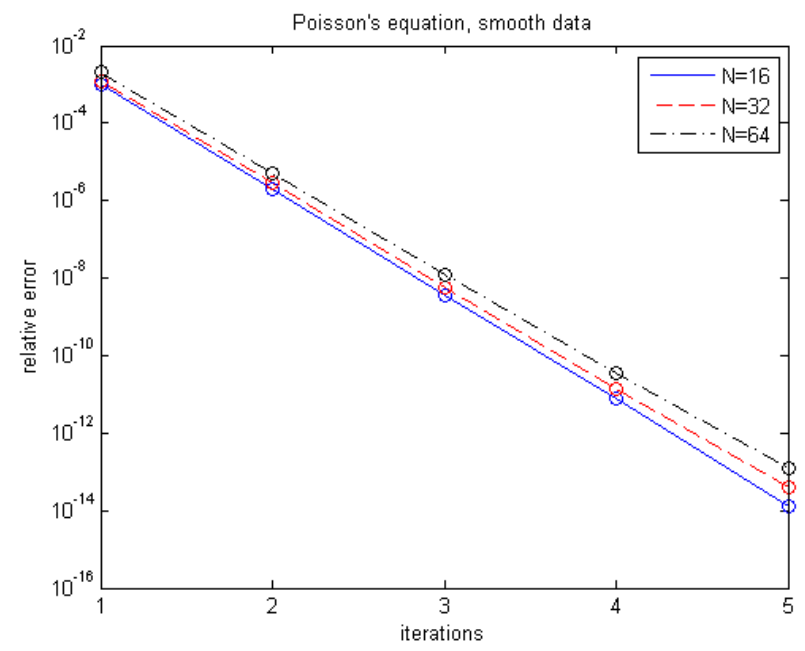

FIGURE 1. Relative error in solutions to Poisson's equation (19), (20), (21) computed by 2-node block KSS methods with residual correction. 
TABLE 1. Relative $L^{2}$ error, excluding truncation of Fourier series, in solutions of (19), (20), (21) with $N$ grid points per dimension. The third column lists the number of iterations of residual correction.

\begin{tabular}{|c|c|c|}
\hline$N$ & Error & Iterations \\
\hline 16 & $1.3 \mathrm{e}-14$ & 4 \\
32 & $4.0 \mathrm{e}-14$ & 4 \\
64 & $1.2 \mathrm{e}-13$ & 4 \\
\hline
\end{tabular}

We now solve (19) with a coefficient and right-hand side that are less smooth, in that their Fourier coefficients have the decay rate of a function with only two continuous derivatives with respect to either $x$ or $y$, compared to four in the previous experiment. Specifically,

$$
\begin{aligned}
p(x, y) \approx & 4.04+0.017 \cos y+0.0052 \sin y+0.0089 \cos 2 y+0.0042 \cos 3 y+ \\
& 0.0021 \sin 3 y+0.029 \cos x+0.014 \sin x+0.0083 \cos (x+y)+ \\
& 0.0036 \cos (x+2 y)+0.0023 \cos (x+3 y)+0.0066 \cos (x-2 y)+0.0073 \cos (x-y)+ \\
& 0.0046 \sin (x-y)+0.0072 \cos 2 x+0.0038 \cos (2 x+y)+0.0018 \sin (2 x+y)+ \\
& 0.004 \cos (2 x-y)-0.0034 \sin (2 x-y)+0.004 \cos 3 x+0.0033 \cos (3 x+y)+ \\
& 0.0026 \cos (3 x-y), \\
g(x, y) \approx & -2.39 \sin y+4.93 \sin 2 y+3.82 \sin 3 y-0.31 \sin x-1.44 \sin (x+y)+ \\
& 0.68 \sin (x+2 y)-1.37 \sin (x+3 y)-0.98 \sin (x-3 y)-5.75 \sin (x-y)- \\
& 1.78 \sin 2 x-1.15 \sin (2 x+y)-1.21 \sin (2 x+2 y)-1.67 \sin (2 x+3 y)- \\
& 0.24 \sin (2 x-3 y)+0.95 \sin (2 x-2 y)-0.12 \cos (2 x-y)-5.47 \sin (2 x-y)+ \\
& 0.34 \cos 3 x+8.84 \sin 3 x+0.19 \cos (3 x+y)+4.95 \sin (3 x+y)+ \\
& 2.3 \sin (3 x+2 y)-1.84 \sin (3 x+3 y)+0.72 \sin (3 x-3 y)+0.79 \sin (3 x-2 y)+ \\
& 0.98 \sin (3 x-y),
\end{aligned}
$$

and with Dirichlet boundary conditions. The results are shown in Figure 2 and Table 2. We observe that even though the Fourier coefficients of the problem data decay more slowly than in the previous problem by two orders of magnitude, the computed solution has comparable accuracy, after just one extra iteration of residual correction. As before, the error increases only moderately as the number of grid points per dimension is doubled.

\section{A Multigrid Approach}

Figure 3 displays the error in solutions to a one-dimensional analogue of (19) with smoothly varying coefficients and data, after each pass of residual correction, using a 2node block KSS method with 256 and 512 grid points, respectively. It can easily be seen 


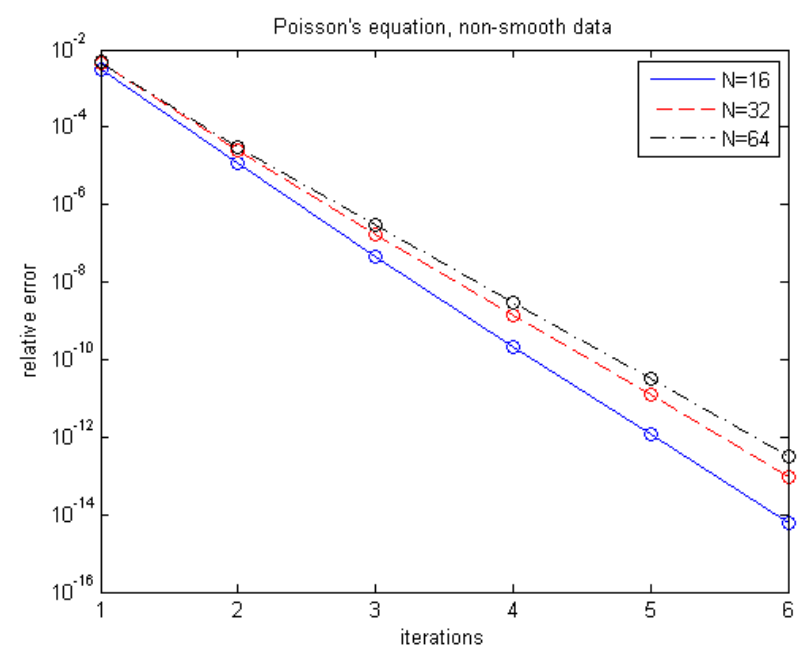

FIGURE 2. Relative error in solutions to Poisson's equation (19), (22), (23) computed by 2-node block KSS methods with residual correction.

TABLE 2. Relative $L^{2}$ error, excluding truncation of Fourier series, in solutions of (19), (22), (23) with $N$ grid points per dimension. The third column lists the number of iterations of residual correction.

\begin{tabular}{|c|c|c|}
\hline$N$ & Error & Iterations \\
\hline 16 & $6.0 \mathrm{e}-15$ & 5 \\
32 & $8.9 \mathrm{e}-14$ & 5 \\
64 & $3.1 \mathrm{e}-13$ & 5 \\
\hline
\end{tabular}

from the figure, and confirmed by a simple Fourier analysis, that for Poisson's equation, the error in the initial iterations of residual correction is smooth, but becomes less smooth as residual correction continues. Furthermore, the initial smooth error is essentially independent of the grid resolution. Therefore, it makes sense to use a multigrid-like approach, in which initial solutions are computed on a coarse grid, and corrected on a finer grid; that is, the opposite sequence of a traditional V-cycle.

We now try this multigrid-like approach on the following one-dimensional problem

$$
\left.\frac{\partial}{\partial x}\left(p(x) \frac{\partial u}{\partial x}\right)=g(x),\right)
$$

with Dirichlet boundary conditions, where

$$
\begin{aligned}
p(x) \approx & 4.2206+0.2436 \cos x+0.036459 \sin x+0.022538 \cos 2 x- \\
& 0.0070943 \sin 2 x+0.0019983 \cos 3 x+0.0011086 \sin 3 x \\
g(x) \approx & 0.010536 \sin x-0.00012202 \cos 2 x-0.0014578 \sin 2 x- \\
& 0.01084 \sin 3 x
\end{aligned}
$$



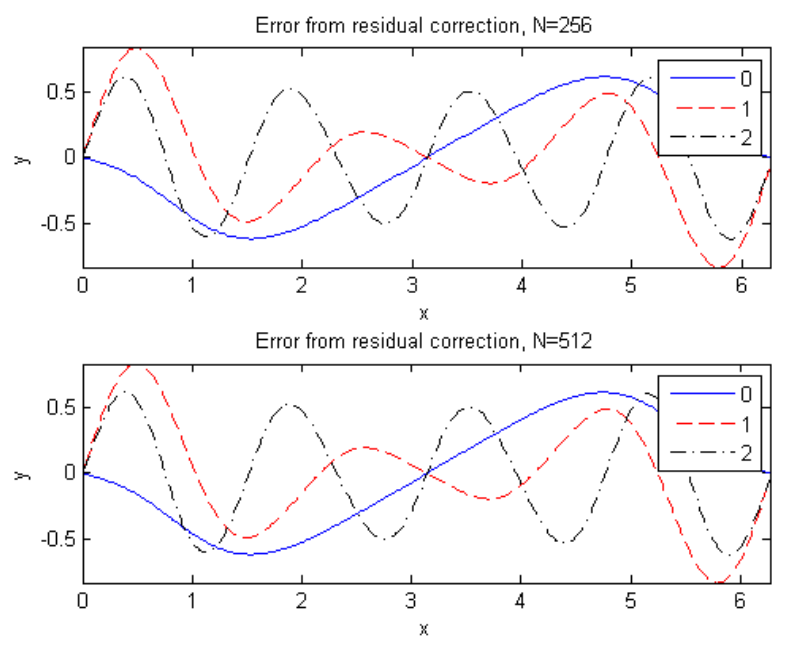

FIGURE 3. Error in computed solutions to a 1-D analogue of (19) after zero (solid blue curve), one (dashed red curve), and two (dotted-dashed black curve) iterations of residual correction in conjunction with a 2-node block KSS method on a 256-point grid (top plot) and a 512-point grid (bottom plot).

The results are shown in Table 3 and Figure 4. The problem is solved in the following ways:

- Performing residual correction on a fine grid containing $N=1024$ grid points

- Performing residual correction on a sequence of grids, beginning with a coarse grid containing only $N=32$ grid points, and then refining to $N=64,128,256,512$, and finally 1024 grid points.

In both cases, a 2-node block KSS method is used, as before. Error is measured by comparing the discrete Fourier transforms of each approximate solution to that of the exact solution on the finest grid. We observe that except for a couple of iterations, the error in the two methods is nearly identical, and in all cases it is comparable, but of course the multigrid scheme is much more efficient due to its use of coarser grids.

\section{The Helmholtz Equation}

Now, we apply a 2-node block KSS method to the inhomogeneous Helmholtz equation

$$
\Delta u(x, y)+k(x, y)^{2} u(x, y)=g(x, y),
$$

with periodic boundary conditions, where

$$
\begin{aligned}
k(x, y)^{2} \approx & 4.0901+0.0054715 \cos y+0.0016952 \sin y+0.0060875 \cos x+ \\
& 0.00039064 \sin x+0.0013668 \cos (x+y)-0.00069206 \sin (x+y)+ \\
& 0.0011185 \cos (x-y) \\
g(x, y) \approx & 0.41002+0.0069992 \cos y-0.0017691 \sin y+0.004967 \cos x+
\end{aligned}
$$


TABLE 3. Relative error in solutions to Poisson's equation (24), (25), (26) computed by 2-node block KSS methods with residual correction, on multiple grids with $N=32,64,128,256,512,1024$ grid points, and a single grid with $N=1024$ grid points.

\begin{tabular}{|c|c|c|c|}
\hline Method & $N$ & Corrections & Error \\
\hline \multirow{5}{*}{ Multigrid } & 32 & 0 & $9.973 \mathrm{e}-3$ \\
& 64 & 1 & $1.271 \mathrm{e}-4$ \\
& 128 & 2 & $2.403 \mathrm{e}-6$ \\
& 256 & 3 & $8.331 \mathrm{e}-8$ \\
& 512 & 4 & $7.228 \mathrm{e}-9$ \\
& 1024 & 5 & $1.182 \mathrm{e}-11$ \\
\hline \multirow{5}{*}{ Fine grid only } & 1024 & 0 & $1.088 \mathrm{e}-2$ \\
& 1024 & 1 & $1.470 \mathrm{e}-4$ \\
& 1024 & 2 & $2.543 \mathrm{e}-6$ \\
& 1024 & 3 & $4.725 \mathrm{e}-8$ \\
& 1024 & 4 & $7.582 \mathrm{e}-10$ \\
& 1024 & 5 & $1.402 \mathrm{e}-11$ \\
\hline
\end{tabular}

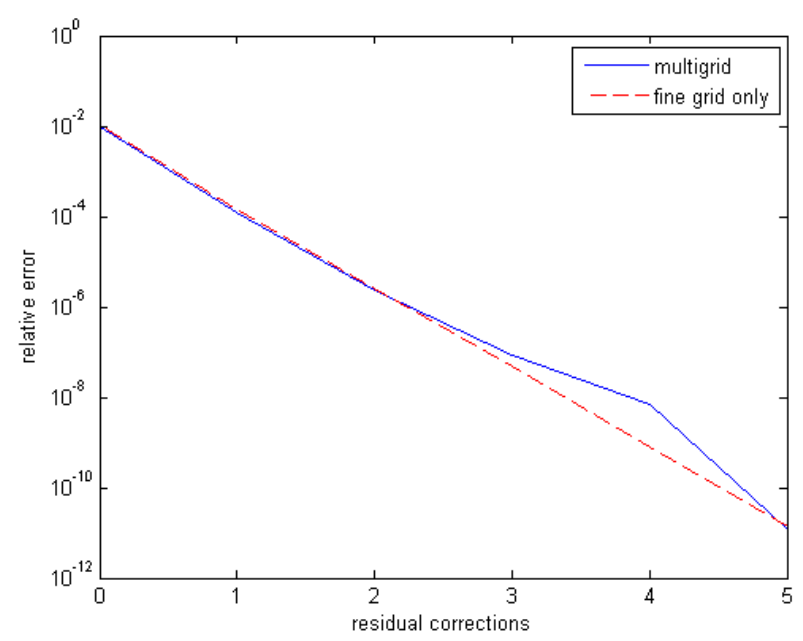

FIGURE 4. Relative error in solutions to Poisson's equation (24), (25), (26) computed by 2-node block KSS methods with residual correction, on multiple grids with $N=32,64,128,256,512,1024$ grid point (blue solid curve) and a single grid with $N=1024$ grid points (red dashed curve).

$$
\begin{aligned}
& 0.0029314 \sin x+0.0025509 \cos (x+y)-0.0001331 \sin (x+y)+ \\
& 0.00069903 \cos (x-y)-0.00059274 \sin (x-y) .
\end{aligned}
$$

The coefficient $k(x, y)^{2}$ is shown in Figure 5(a). The results are shown in Table 4 and Figure 6(a). We observe even more accuracy than in the previous experiment for Poisson's equation, and even more agreement in the error between the multigrid and single-grid methods.

Generally, the dominant portion of the error arises from the computation of the Fourier 
(a)

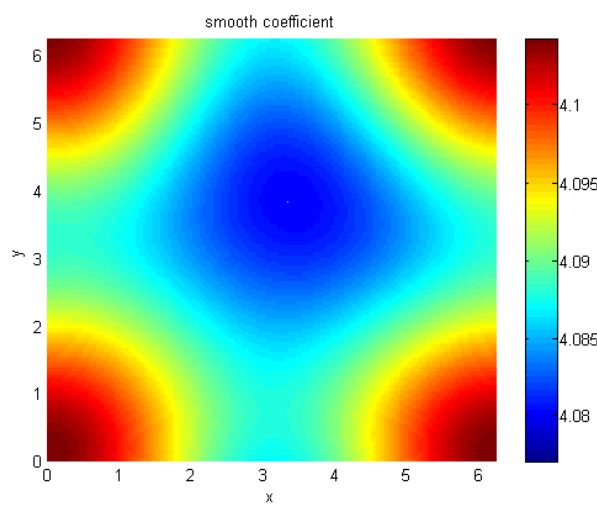

(b)

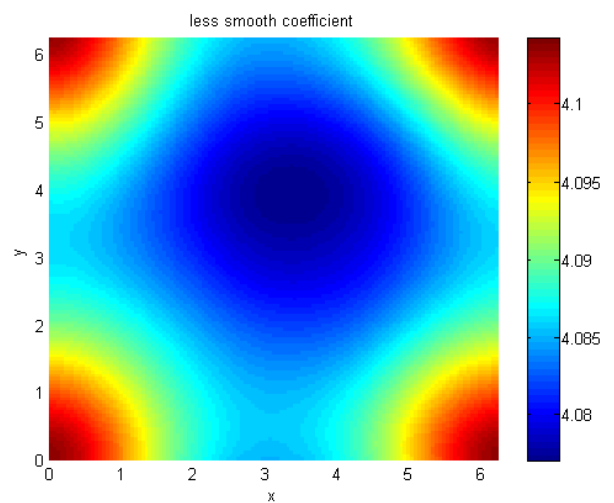

FIGURE 5. (a) Left plot: smooth coefficient $k(x, y)^{2}$ in (27) and (30). (b) Right plot: less smooth coefficient $k(x, y)^{2}$ in (27) and (30).

coefficients corresponding to the region of phase space where the symbol of $L=\Delta+k^{2}$ is smallest. This leads to Gaussian quadrature nodes near the singularity in the integrand $f(\lambda)=\lambda^{-1}$. The integrand is more difficult to approximate accurately by polynomial interpolation near this singularity, and the resulting error is negligibly impacted by grid refinement.

However, this error is substantially reduced if the coefficient $k(x, y)^{2}$ and right-hand side $g(x, y)$ are very smooth, because then the basis functions $e^{i \omega \cdot \mathbf{x}}$ are nearly eigenfunctions, which makes most of the terms $\alpha_{j} \beta_{j}$ in (8) negligibly small. Future work will explore the use of preconditioning similarity transformations, aided by fast algorithms presented in [3] for application of Fourier integral operators, for homogenizing variable coefficients in order to improve the performance of KSS methods for such problems.

TABLE 4. Relative error in solutions to Poisson's equation (27), (28), (29) computed by 2-node block KSS methods with residual correction, on multiple grids with $N=8,16,32,64,128$ grid points per dimension, and a single grid with $N=128$ grid points per dimension.

\begin{tabular}{|c|c|c|c|}
\hline Method & $N$ & Corrections & Error \\
\hline \multirow{5}{*}{ Multigrid } & 8 & 0 & $1.590 \mathrm{e}-5$ \\
& 16 & 1 & $1.605 \mathrm{e}-7$ \\
& 32 & 2 & $3.369 \mathrm{e}-9$ \\
& 64 & 3 & $3.844 \mathrm{e}-11$ \\
& 128 & 4 & $7.634 \mathrm{e}-13$ \\
\hline \multirow{5}{*}{ Fine grid only } & 128 & 0 & $1.590 \mathrm{e}-5$ \\
& 128 & 1 & $1.014 \mathrm{e}-7$ \\
& 128 & 2 & $4.764 \mathrm{e}-9$ \\
& 128 & 3 & $3.626 \mathrm{e}-11$ \\
& 128 & 4 & $9.992 \mathrm{e}-13$ \\
\hline
\end{tabular}

Next, we solve the modified problem

$$
\Delta u(x, y)+100 k(x, y)^{2} u(x, y)=g(x, y),
$$


(a)

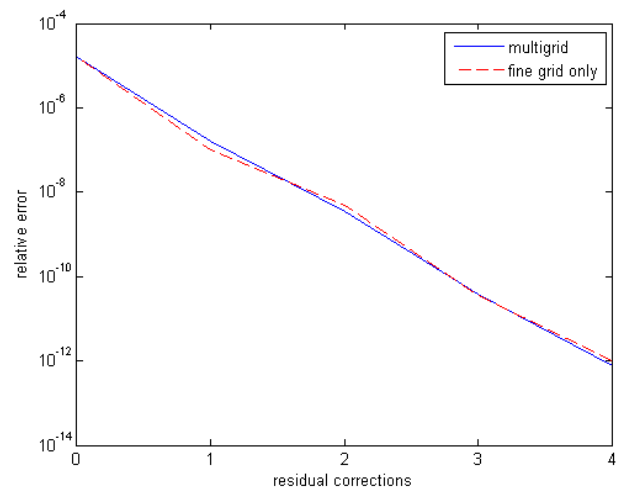

(b)

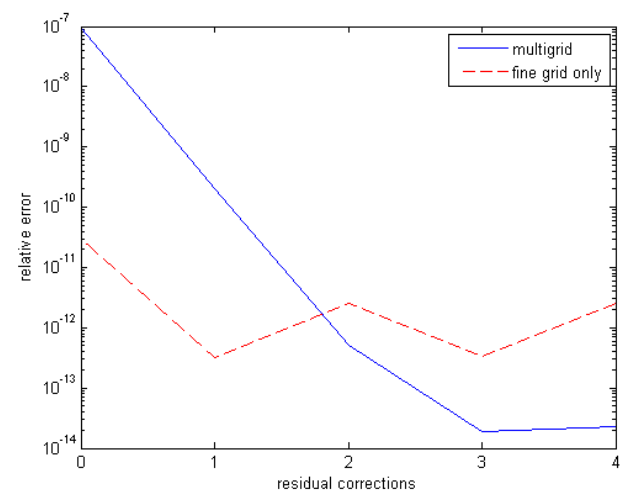

FIGURE 6. (a) Left plot: Relative error in solutions to the Helmholtz equation (27), (28), (29) computed by 2-node block KSS methods with residual correction, on multiple grids with $N=8,16,32,64,128$ grid points per dimension (blue solid curve) and a single grid with $N=128$ grid points per dimension (red dashed curve). (b) Right plot: Relative error in solutions to the Helmholtz equation (30), (28), (29) computed by 2-node block KSS methods with residual correction, on multiple grids with $N=$ $8,16,32,64,128$ grid points per dimension (blue solid curve) and a single grid with $N=128$ grid points per dimension (red dashed curve).

with periodic boundary conditions and $k(x, y)$ and $g(x, y)$ as defined in (28), (29). The results are shown in Table 5 and Figure 6(b). We see that even though there is a greater degree of indefiniteness in the operator $L$, the error in the multigrid method is even less than with the smaller coefficient. In fact, high accuracy is achieved after only a single residual correction. This is because the dominant portion of the error, described earlier, corresponds to Fourier coefficients that, in the exact solution, are significantly smaller. On the other hand, the single-grid approach, while immediately yielding high accuracy, is not aided by residual correction. This is because residual correction requires a particularly accurate residual, but at this level of accuracy, and with no finer grid available, a sufficiently accurate residual cannot be computed.

TABLE 5. Relative error in solutions to the Helmholtz equation (30), (28), (29) computed by 2-node block KSS methods with residual correction, on multiple grids with $N=8,16,32,64,128$ grid points per dimension, and a single grid with $N=128$ grid points per dimension.

\begin{tabular}{|c|c|c|c|}
\hline Method & $N$ & Corrections & Error \\
\hline \multirow{5}{*}{ Multigrid } & 8 & 0 & $9.797 \mathrm{e}-8$ \\
& 16 & 1 & $2.033 \mathrm{e}-10$ \\
& 32 & 2 & $5.181 \mathrm{e}-13$ \\
& 64 & 3 & $1.912 \mathrm{e}-14$ \\
& 128 & 4 & $2.268 \mathrm{e}-14$ \\
\hline \multirow{5}{*}{ Fine grid only } & 128 & 0 & $3.036 \mathrm{e}-11$ \\
& 128 & 1 & $3.219 \mathrm{e}-13$ \\
& 128 & 2 & $2.586 \mathrm{e}-12$ \\
& 128 & 3 & $3.309 \mathrm{e}-13$ \\
& 128 & 4 & $2.568 \mathrm{e}-12$ \\
\hline
\end{tabular}


We now solve (27) with less smooth coefficients and data,

$$
\begin{aligned}
k(x, y)^{2} \approx & 4.0867+0.0054715 \cos y+0.0016952 \sin y+0.00060255 \cos 2 y+ \\
& 0.0060875 \cos x+0.00039064 \sin x+0.0013668 \cos (x+y)- \\
& 0.00069206 \sin (x+y)+0.00023799 \cos (x+2 y)+ \\
& 0.00013712 \cos (x-2 y)-0.00013463 \sin (x-2 y)+ \\
& 0.0011185 \cos (x-y)+0.00072674 \cos 2 x+ \\
& 0.00031291 \cos (2 x+y)+0.00021032 \cos (2 x-y) \\
g(x, y) \approx & 0.41086+0.0069992 \cos y-0.0017691 \sin y+0.00014033 \cos 2 y+ \\
& 0.004967 \cos x+0.0029314 \sin x+0.0025509 \cos (x+y)- \\
& 0.0001331 \sin (x+y)+0.00033062 \cos (x+2 y)+ \\
& 0.00012874 \cos (x-2 y)+0.00069903 \cos (x-y)- \\
& 0.00059274 \sin (x-y)+0.00081406 \cos 2 x- \\
& 0.00025677 \sin 2 x+0.00012879 \cos (2 x+y)+ \\
& 0.00018624 \sin (2 x+y)+0.00011291 \cos (2 x-y)+ \\
& 0.00020258 \sin (2 x-y)+0.00012862 \cos 3 x
\end{aligned}
$$

and with periodic boundary conditions. The coefficient $k(x, y)^{2}$ is shown in Figure 5(b). The results are shown in Table 6 and Figure 7(a). We observe that the reduced smoothness leads to some loss of accuracy, but the multigrid and single-grid methods still perform comparably. Furthermore, the errors are also comparable to that achieved for Poisson's equation, for which coefficients and data of the same smoothness were used.

TABLE 6. Relative error in solutions to the Helmholtz equation (27), (31), (32) computed by 2-node block KSS methods with residual correction, on multiple grids with $N=8,16,32,64,128$ grid points per dimension, and a single grid with $N=128$ grid points per dimension.

\begin{tabular}{|c|c|c|c|}
\hline Method & $N$ & Corrections & Error \\
\hline \multirow{5}{*}{ Multigrid } & 8 & 0 & $3.488 \mathrm{e}-4$ \\
& 16 & 1 & $8.434 \mathrm{e}-6$ \\
& 32 & 2 & $7.246 \mathrm{e}-7$ \\
& 64 & 3 & $6.118 \mathrm{e}-8$ \\
& 128 & 4 & $2.356 \mathrm{e}-11$ \\
\hline \multirow{5}{*}{ Fine grid only } & 128 & 0 & $3.815 \mathrm{e}-4$ \\
& 128 & 1 & $1.166 \mathrm{e}-5$ \\
& 128 & 2 & $4.172 \mathrm{e}-8$ \\
& 128 & 3 & $1.035 \mathrm{e}-8$ \\
& 128 & 4 & $6.794 \mathrm{e}-11$ \\
\hline
\end{tabular}

Finally, we solve the same problem, except that the coefficient $k^{2}$ is replaced by $100 k^{2}$. The results are shown in Table 7 and Figure 7(b). We see that the combination of reduced smoothness and the magnitude of the coefficient poses difficulty for block KSS methods as the number of grid points increases. Due to the reduced smoothness, the dominant 
(a)

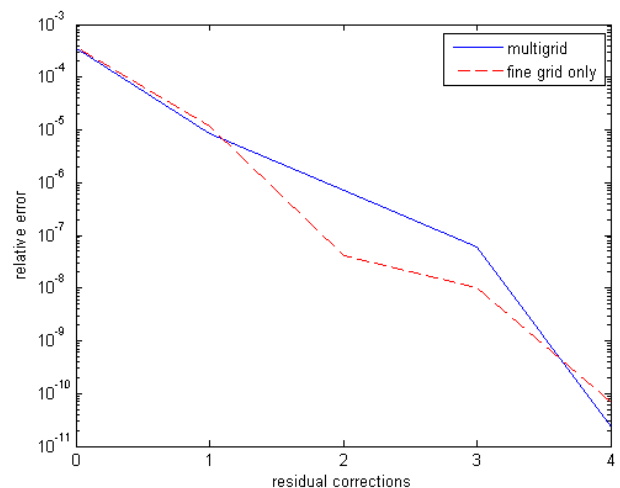

(b)

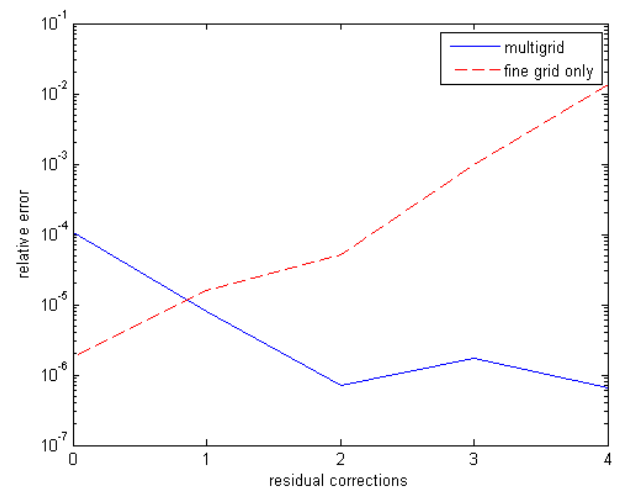

FIGURE 7. (a) Right plot: Relative error in solutions to the Helmholtz equation (27), (31), (32) computed by 2-node block KSS methods with residual correction, on multiple grids with $N=8,16,32,64,128$ grid points per dimension (blue solid curve) and a single grid with $N=128$ grid points per dimension (red dashed curve). (b) Left plot: Relative error in solutions to the Helmholtz equation (30), (31), (32) computed by 2-node block KSS methods with residual correction, on multiple grids with $N=8,16,32,64,128$ grid points per dimension (blue solid curve) and a single grid with $N=128$ grid points per dimension (red dashed curve).

portion of the error corresponds to Fourier coefficients that are more significant in the exact solution. Furthermore, because these Fourier coefficients correspond to higher frequencies than when $k^{2}$ is relatively small, higher-frequency oscillations are introduced, which are then amplified by differentiation during the computation of the recursion coefficients in $\mathscr{T}_{K}$, resulting in larger errors. In fact, the accuracy degrades when residual correction is applied on a single fine grid, due to the aforementioned inability to obtain an accurate residual, but the multigrid approach is still effective to reducing error, to at least some extent.

TABLE 7. Relative error in solutions to the Helmholtz equation (30), (31), (32) computed by 2-node block KSS methods with residual correction, on multiple grids with $N=8,16,32,64,128$ grid points per dimension, and a single grid with $N=128$ grid points per dimension.

\begin{tabular}{|c|c|c|c|}
\hline Method & $N$ & Corrections & Error \\
\hline \multirow{5}{*}{ Multigrid } & 8 & 0 & $1.077 \mathrm{e}-4$ \\
& 16 & 1 & $7.919 \mathrm{e}-6$ \\
& 32 & 2 & $7.200 \mathrm{e}-7$ \\
& 64 & 3 & $1.753 \mathrm{e}-6$ \\
& 128 & 4 & $6.623 \mathrm{e}-7$ \\
\hline \multirow{5}{*}{ Fine grid only } & 128 & 0 & $1.755 \mathrm{e}-6$ \\
& 128 & 1 & $1.569 \mathrm{e}-5$ \\
& 128 & 2 & $5.172 \mathrm{e}-5$ \\
& 128 & 3 & $9.732 \mathrm{e}-4$ \\
& 128 & 4 & $1.376 \mathrm{e}-2$ \\
\hline
\end{tabular}




\section{SUMMARY AND FUTURE WORK}

We have demonstrated that KSS methods, while originally designed for time-dependent PDE, can also be applied to time-independent elliptic PDE with smoothly varying coefficients. Using residual correction on successively finer grids within a multigridlike framework, these methods can compute highly accurate solutions, even for the Helmholtz equation, for which the integrand in the Riemann-Stieltjes integrals used to compute Fourier coefficients is singular.

Future work will extend the approach described in this paper to problems in which

- the coefficients and data are oscillatory or discontinuous. Recent work, described in [14], employs the polar decomposition (12) to alleviate difficulties caused by such coefficients or data by causing cancellation of spurious high-frequency oscillations. Our goal is to combine this approach with block KSS methods in order to generalize the superior accuracy of the block approach to these more difficult problems. Alternative approaches may benefit from reprojection techniques (see, for example, [4]) or grid adapation (see [18]).

- the domain has a complicated geometry. The main ideas behind block KSS methods are unrelated to the choice of a Fourier basis; they can be applied to any Galerkin method. Ongoing work considers the application of KSS methods to approximating functions of stiffness matrices arising from finite element discretizations. Another avenue of extension builds on Fourier continuation along lines, as used by Bruno and Lyon [2].

In addition, we will consider the use of Gauss-Radau and Gauss-Lobatto rules, in which selected nodes are prescribed, to deal with the singularity associated with the Helmholtz equation.

While the experiments in this paper pertaining to Poisson's equation used homogeneous Dirichlet boundary conditions, periodic boundary conditions can easily be used instead, as demonstrated in the examples for the Helmholtz equation, since the eigenfunction corresponding to the zero eigenvalue in (19) is known. This problem is of particular interest in the study of mercury emissions by coal-fired power plants, and their interaction with activated carbon and fly ash, because the intermolecular potentials of reactants and products are needed to compute thermodynamic properties in the simulation of power plant conditions. Future work will include the incorporation of block KSS methods into a modified particle-particle, particle-mesh $\left(P^{3} M\right)$ algorithm [10] for this application.

\section{REFERENCES}

1. K. Atkinson, An Introduction to Numerical Analysis, 2nd Ed. (1989) Wiley.

2. O. Bruno and M. Lyon, "High-order unconditionally-stable FC-AD solvers for general smooth domains I. Basic elements", submitted.

3. E. Candes, L. Demanet, and L. Ying, "Fast Computation of Fourier Integral Operators", SIAM J. Sci. Comput. 29(6) (2007), p. 2464-2493.

4. Gelb, A., Tanner, J.: Robust Reprojection Methods for the Resolution of the Gibbs Phenomenon. Appl. Comput. Harmon. Anal. 20 (2006) 3-25. 
5. G. H. Golub and C. Meurant, "Matrices, Moments and Quadrature", in Proceedings of the 15th Dundee Conference, June-July 1993, D. F. Griffiths and G. A. Watson (eds.), Longman Scientific \& Technical, 1994.

6. G. H. Golub and R. Underwood, "The block Lanczos method for computing eigenvalues", Mathematical Software III, J. Rice Ed., (1977), p. 361-377.

7. G. H. Golub and J. Welsch, "Calculation of Gauss Quadrature Rules", Math. Comp. 23 (1969), p. 221-230.

8. P. Guidotti, J. V. Lambers, and K. Sølna, "Analysis of Wave Propagation in 1D Inhomogeneous Media”, Numer. Funct. Anal. Optim 27 (2006), p. 25-55.

9. M. Hochbruck and C. Lubich, "On Krylov Subspace Approximations to the Matrix Exponential Operator", SIAM J. Numer. Anal. 34 (1996), p. 1911-1925.

10. R. W. Hockney, S. P. Goel, and J. W. Eastwood, "A 10,000 particle molecular dynamics model with long range forces", Chemical Physics Letters, 21(3) (1973), p. 589-591.

11. J. V. Lambers, "Derivation of High-Order Spectral Methods for Time-Dependent PDE Using Modified Moments", Electr. Trans. Num. Anal. 28 (2007), p. 114-135.

12. J. V. Lambers, "Enhancement of Krylov Subspace Spectral Methods by Block Lanczos Iteration", Electronic Transactions on Numerical Analysis 31 (2008), p. 86-109.

13. J. V. Lambers, "An Explicit, Stable, High-Order Spectral Method for the Wave Equation Based on Block Gaussian Quadrature", IAENG Journal of Applied Mathematics 38 (2008), p. 333-348.

14. J. V. Lambers, "Krylov Subspace Spectral Methods for the Time-Dependent Schrödinger Equation with Non-Smooth Potentials", Num. Alg. 51 (2009), p. 349-380.

15. J. V. Lambers, "Krylov Subspace Spectral Methods for Variable-Coefficient Initial-Boundary Value Problems", Electron. Trans. Numer. Anal. 20 (2005), p. 212-234.

16. J. V. Lambers, "Practical Implementation of Krylov Subspace Spectral Methods", J. Sci. Comput. 32 (2007), p. 449-476.

17. J. V. Lambers, "A Spectral Time-Domain Method for Computational Electrodynamics", Proceedings of the IAENG Multiconference of Engineers and Computer Scientists (2009), p. 2111-2116.

18. T. Vallius and M. Honkanen, "Reformulation of the Fourier nodal method with adaptive spatial resolution: application to multilevel profiles", Opt. Expr. 10(1) (2002), p. 24-34. 